\title{
Regularization for effective field theory with two heavy particles ${ }^{1}$
}

\author{
J. L. Goity, ${ }^{\mathrm{a}, \mathrm{b}}$ D. Lehmann, ${ }^{\mathrm{a}, \mathrm{b}}$ G. Prézeau, ${ }^{\mathrm{a}, 2}$ and J. Saez ${ }^{\mathrm{a}}$ \\ ${ }^{a}$ Department of Physics, Hampton University, Hampton, VA 23668, USA \\ b Thomas Jefferson National Accelerator Facility, \\ 12000 Jefferson Avenue M.S. 12H2, Newport News, VA 23606, USA
}

\begin{abstract}
A regularization for effective field theory with two propagating heavy particles is constructed. This regularization preserves the low-energy analytic structure, implements a low-energy power counting for the one-loop diagrams, and preserves symmetries respected by dimensional regularization.
\end{abstract}

Key words: Regularization, Effective Field Theory, Baryon Chiral Perturbation Theory, NN Effective Field Theory, Low-Energy Power Counting.

PACS: 11.10.-z, 11.15.Bt, 12.39.Hg, 12.39.Fe

\section{Introduction}

One of the necessary ingredients in an effective field theory (EFT) is the ultraviolet regularization. A natural regularization for the EFT is one that removes from loop integrals all contributions with momenta above the EFT energy scale, $\Lambda_{\mathrm{EFT}}$, and that also implements a low-energy counting by making the small energy scales in the EFT, namely small momenta and light particle masses, the scales that set the magnitude of power ultraviolet divergences. One example of such a natural regularization is dimensional regularization in EFT with only light particles, such as Chiral Perturbation Theory (ChPT) [1,2] for quasi-Goldstone pions. In dimensional regularization the scale of $\mathrm{ChPT}$ is given in terms of the pion decay constant $F_{\pi}$, and power divergences of loop

1 JLAB-THY-00-46

2 Mail address: Thomas Jefferson National Accelerator Facility, 12000 Jefferson Avenue M.S. 12H2, Newport News, VA 23606, USA 
integrals are proportional to the small scales $\left(M_{\pi}^{2}\right.$ and/or invariants formed with the small external momenta) divided by the appropriate powers of $F_{\pi}$. Although a momentum cutoff can be used, it does not deal well with power divergences, as these contain pieces proportional to powers of the cutoff. In this case, an EFT can also be formulated, but it is more complicated to deal with because the low-energy power counting must be recovered in the process of renormalization.

In an EFT with a heavy stable particle, such as ChPT with nucleons, the heavy particle mass $M$ is a new scale in the theory (some heavy masses could be much larger than $\Lambda_{\mathrm{EFT}}$, e.g., baryon masses in large- $N_{\mathrm{c}}$ QCD). With the inclusion of $M$, dimensional regularization ceases to be a natural regularization. Indeed, the power divergences lead now to contributions proportional to fractional powers of $M$, i.e. contributions that give rise to terms that are powers of $M^{2}$ times $\log M^{2}$ in $d \rightarrow 4$ dimensions. One such example is the nucleon self-energy in ChPT calculated in dimensional regularization [3]. Although the EFT can be consistently regularized with dimensional regularization, a natural regularization is more convenient.

In the case, where a single heavy particle propagates through a Feynman diagram, one way to remove fractional powers of $M[7]$ is to perform an expansion in $1 / M$ at the level of the Lagrangian [4], eliminating in this way $M$ from the propagators. This approach implies that all the Green functions must be analytic functions of $1 / M$ in the low-energy region. This, however, is not correct because there are low-energy singularities in $1 / M$, such as the anomalous thresholds (e.g., in the scalar form factor of the nucleon [5]), which the EFT must retain. Recently, a regularization based on dimensional regularization that avoids these problems of the $1 / M$ expansion was developed. Building on work by Ellis and Tang [6], Becher and Leutwyler [7] developed a regularization scheme that is natural, preserves the low-energy structure and internal symmetries, and is Lorentz covariant. This regularization has been called infrared regularization.

In the case of two heavy particles, the $1 / M$ expansion cannot be applied due to the two-heavy particle threshold in the s-channel. It is, therefore, very important to develop a natural regularization for this case as well. In this letter such a regularization is developed along lines similar to those of the infrared regularization [7]. Starting from standard dimensional regularization, a modification of the Feynman parameter integration domain is introduced leading to a natural regularization. Its implementation is presented in a Lorentz covariant framework for basic one-loop diagrams. 


\section{Regularization of one-loop diagrams}

In this section, the basic one-loop diagrams in the two-heavy-particle sector are discussed: these are the bubble diagram Fig. 1, the triangle diagram Fig. 2 and the box diagram Fig. 4. In each case, the dimensionally regularized Feynman integral $I$ can be decomposed into

$$
I=\bar{I}+R
$$

where the fractional powers of $M$ are entirely contained in $R$. In addition, $R$ is an analytic function in the low-energy domain (i.e. local) and is referred to as the regular part. The low-energy part $\bar{I}$ contains all the low-energy analytic structure of the integral. Because $\bar{I}$ does not contain any fractional powers of $M$, it satisfies a low-energy power counting as discussed in Sect. 3. The loop integral in the EFT Dimensional Regularization (EFTDR) is then identified with $\bar{I}$.

The low-energy domain is expressed in terms of the heavy mass $M$, the light mass $m$, and the invariants $P_{i} \cdot P_{j}$ formed with the external heavy particle momenta, and is defined by the conditions $\left|P_{i} \cdot P_{j}-M^{2}\right| \ll \Lambda_{\mathrm{EFT}}^{2}$ and $m \ll$ $\Lambda_{\mathrm{EFT}}$. As in ChPT, low energy quantities such as $m$, external momenta of light particles, differences of external momenta of heavy particles, etc., will be said to be of order $p$.

Although integrals that carry factors of the loop momentum in the numerator are not explicitly presented here, such integrals can be reduced to combinations of scalar integrals of the form discussed here, to integrals that involve only one heavy propagator that can be regularized using the infrared regularization, and to integrals that involve only light propagators.

\subsection{The bubble diagram}

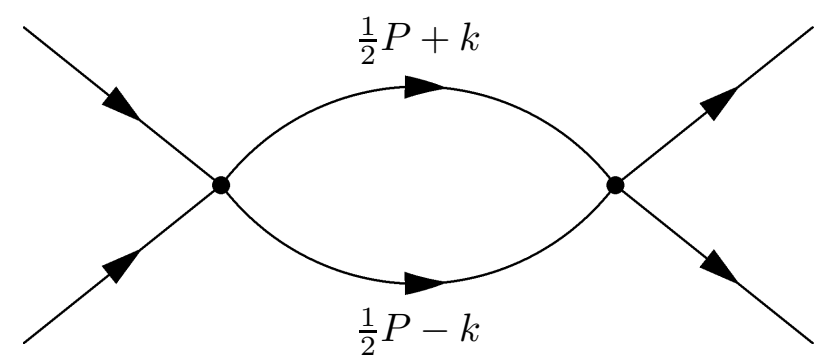

Fig. 1.

The simplest diagram in the two-heavy-particle sector is the bubble diagram of Fig. 1 with the two heavy particles propagating in the s-channel. The scalar integral for this diagram is 


$$
I_{2}\left(s, M^{2}\right)=\mu^{4-d} \int \frac{\mathrm{d}^{d} k}{(2 \pi)^{d}} \frac{\mathrm{i}}{\left(\frac{P}{2}-k\right)^{2}-M^{2}+\mathrm{i} \epsilon} \frac{\mathrm{i}}{\left(\frac{P}{2}+k\right)^{2}-M^{2}+\mathrm{i} \epsilon},
$$

where $s \equiv P^{2}$ and $P$ is the total incoming momentum. Combining the propagators using a Feynman parameter integral, the integration over momentum yields

$$
I_{2}=\kappa_{2} \int_{-1}^{1} \mathrm{~d} w\left[\frac{s}{4}\left(w^{2}-1\right)+M^{2}-\mathrm{i} \epsilon\right]^{d / 2-2}
$$

with

$$
\kappa_{2} \equiv-\frac{\mathrm{i}}{2} \frac{1}{(4 \pi)^{2}} \Gamma\left(2-\frac{d}{2}\right)\left(4 \pi \mu^{2}\right)^{2-d / 2}
$$

This integral has a pinch singularity at $w=0$ when $s=s_{+} \equiv 4 M^{2}$, which is the branch point of the two-heavy-particle cut. The integration boundaries give rise to fractional powers of $M$ which spoil low-energy power counting. This can be shown below threshold by scaling the small quantity $\left(M^{2}-s / 4\right)$ out of Eq. (3):

$$
I_{2}=\kappa_{2}\left(\frac{s}{4}\right)^{d / 2-2} \alpha^{d-3} \int_{-\frac{1}{\alpha}}^{\frac{1}{\alpha}} \mathrm{d} w\left(w^{2}+1\right)^{d / 2-2},
$$

where

$$
\alpha \equiv \sqrt{\frac{s_{+}-s-\mathrm{i} \epsilon}{s}}
$$

is a small quantity in the low-energy domain which characterizes the infrared behavior of the bubble diagram. From Eq. (5) it is clear that only the integral limits can change the power of $\alpha$.

The integral can then be separated into its regular and low-energy part:

$$
I_{2}=\bar{I}_{2}+R_{2}
$$

with

$$
\bar{I}_{2} \equiv \kappa_{2}\left(\frac{s}{4}\right)^{d / 2-2} \alpha^{d-3} \int_{-\infty}^{\infty} \mathrm{d} w\left(w^{2}+1\right)^{d / 2-2}
$$

$\bar{I}_{2}$ and $I_{2}$ have the same low-energy analytic structure as can be verified for $d \rightarrow 4$, where 


$$
I_{2}=\frac{\mathrm{i}}{16 \pi^{2}}\left[-\Gamma\left(2-\frac{d}{2}\right)+\log \left(\frac{M^{2}}{4 \pi \mu^{2}}\right)-2+2 \alpha \arctan \left(\frac{1}{\alpha}\right)\right],
$$

and where, after performing one integration by parts,

$$
\bar{I}_{2}=\frac{\mathrm{i}}{16 \pi} \alpha
$$

$\bar{I}_{2}$ is, therefore, given by the term of order $\alpha$ in the Taylor expansion of Eq. (9) in powers of $\alpha$, as can be immediately seen from

$$
\alpha \arctan \left(\frac{1}{\alpha}\right)=\frac{\pi}{2} \alpha-\alpha \arctan \alpha
$$

The term $\alpha \arctan \alpha$ is analytic in $s_{+}-s$ in the low-energy region. It is then clear that $\bar{I}_{2}$ and $I_{2}$ have the same low energy analytic structure.

In arbitrary d-dimensions, $\bar{I}_{2}$ is proportional to $\left(s_{+}-s\right)^{(d-3) / 2} / \sqrt{s}$, which implies that it does not contain fractional powers of $M$. On the other hand, for $d \rightarrow 4, R_{2}$ is given by

$$
R_{2}=2 \frac{M^{d-4}}{d-3} \kappa_{2}-\frac{\mathrm{i}}{8 \pi^{2}} \alpha \arctan \alpha
$$

while in the low-energy domain and for arbitrary $d$

$$
R_{2}=2 \kappa_{2} M^{d-4} \sum_{m, n=0}^{\infty} c_{m, n} \frac{\left(s_{+}-s\right)^{m+n}}{s_{+}^{m} s^{n}},
$$

where

$$
c_{m, n} \equiv \frac{(-1)^{m} \Gamma^{2}\left(\frac{d}{2}-1\right)}{(d-3-2 n) m ! n ! \Gamma\left(\frac{d}{2}-1-m\right) \Gamma\left(\frac{d}{2}-1-n\right)},
$$

which shows that $R_{2}$ is given by terms analytic in $s-s_{+}$multiplied by a fractional power of $M$. In particular, this shows that the analytic terms not included in $\bar{I}_{2}$ are all of fractional-power type.

The separation into a low-energy part and a regular part with the desired properties has been achieved. Indeed, from Eqs. (6) and (10) it is seen that $\bar{I}_{2}$ has two cuts: a cut due to the factor $\sqrt{s_{+}-s}$ starting at $s=s_{+}$and a cut along the negative axis ending at $s=0$ due to the factor of $1 / \sqrt{s}$. The discontinuity of $I_{2}$ and $\bar{I}_{2}$ across the cut on the positive $s$-axis are equal, and therefore $\bar{I}_{2}$ reproduces the low energy analytic structure of $I_{2}$. The cut along 
the negative $s$-axis is located far outside the low-energy region. Moreover, $\bar{I}_{2}$ does not carry any fractional power of $M$. On the other hand, $R_{2}$ is purely of fractional power type, and has only the cut along the negative $s$-axis.

\subsection{The triangle diagram}

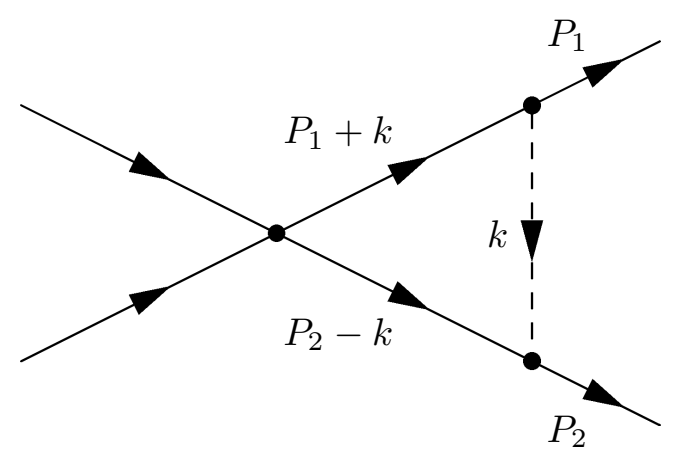

Fig. 2. Triangle diagram in the two heavy-particle-sector. The solid line represents the heavy particle, the dashed line the light particle.

The next diagram to be considered is the triangle diagram shown in Fig. 2 with two heavy particles propagating in the $s$-channel and interacting via a contact interaction and the exchange of a light particle of mass $m$.

The scalar integral for this diagram is

$$
\begin{aligned}
& I_{3}\left(s, P_{1}^{2}, P_{2}^{2}, m^{2}, M^{2}\right) \\
& \quad=\mu^{4-d} \int \frac{\mathrm{d}^{d} k}{(2 \pi)^{d}} \frac{\mathrm{i}}{\left(P_{1}+k\right)^{2}-M^{2}+\mathrm{i} \epsilon} \frac{\mathrm{i}}{\left(P_{2}-k\right)^{2}-M^{2}+\mathrm{i} \epsilon} \frac{\mathrm{i}}{k^{2}-m^{2}+\mathrm{i} \epsilon} .
\end{aligned}
$$

Combining the heavy propagators with a symmetric Feynman parameter integral,

$$
\frac{1}{A_{1} A_{2}}=\frac{1}{2} \int_{-1}^{1} \frac{\mathrm{d} w}{\left(\frac{1+w}{2} A_{1}+\frac{1-w}{2} A_{2}\right)^{2}}
$$

and subsequently combining this result with the light propagator using

$$
\frac{1}{a A^{2}}=2 \int_{0}^{1} \frac{z \mathrm{~d} z}{((1-z) a+z A)^{3}}
$$


the momentum integration yields

$$
\begin{aligned}
I_{3}= & \kappa_{3} \int_{0}^{1} \mathrm{~d} z \int_{-1}^{1} \mathrm{~d} w z[C(w, z)-\mathrm{i} \epsilon]^{d / 2-3}, \\
\kappa_{3}= & -\frac{1}{16 \pi^{2}} \frac{1}{2} \Gamma\left(3-\frac{d}{2}\right)\left(4 \pi \mu^{2}\right)^{2-d / 2}, \\
C(w, z)= & (1-z) m^{2}+z M^{2}-z^{2}\left(1-w^{2}\right) \frac{s}{4} \\
& \quad-z(1-z) \frac{1}{2}\left(P_{1}^{2}+P_{2}^{2}\right)-w z(1-z) \frac{1}{2}\left(P_{1}^{2}-P_{2}^{2}\right) .
\end{aligned}
$$

In order to decompose $I_{3}$ into its low-energy and regular parts,

$$
I_{3}=\bar{I}_{3}+R_{3}
$$

the integration range in $w$ is first extended to the entire real axis in analogy to the bubble diagram,

$$
\bar{I}_{3 \mathrm{~A}}=\kappa_{3} \int_{0}^{1} \mathrm{~d} z \int_{-\infty}^{\infty} \mathrm{d} w z[C(w, z)-\mathrm{i} \epsilon]^{d / 2-3} .
$$

$\bar{I}_{3 \mathrm{~A}}$ contains the proper singularity structure associated with the two-heavyparticle threshold at $s=s_{+}$but does not contain the two-particle cuts corresponding to the heavy-light thresholds at $P_{i}^{2}=(M+m)^{2},(i=1,2)$. All this becomes clear by solving the corresponding Landau equation 3 that determine the location of the singularities in the $(w, z)$-parameter plane shown in Fig. 3. The two-particle thresholds are endpoint singularities, i.e., they lie on the boundary of the original integration domain $[-1,1] \times[0,1]$. The twoheavy-particle cut (indicated by $\times$ in Fig. 3) occurs at $(0,1)$ and is unaffected by extending the domain as in Eq. (20). On the other hand, the heavy-light thresholds (indicated by $\bullet$ ) are located at $\left(+1, \frac{m}{M+m}\right)$ and $\left(-1, \frac{m}{M+m}\right)$, and are lost. The heavy-light thresholds, indeed the full low-energy analytic structure, are restored by defining the low-energy part as

$$
\bar{I}_{3}=\bar{I}_{3 \mathrm{~A}}+\bar{I}_{3 \mathrm{~B}}
$$

with

$$
\bar{I}_{3 \mathrm{~B}}=-\kappa_{3} \int_{0}^{\infty} \mathrm{d} z\left(\int_{-\infty}^{-1} \mathrm{~d} w+\int_{1}^{\infty} \mathrm{d} w\right) z[C(w, z)-\mathrm{i} \epsilon]^{d / 2-3} .
$$

It is evident from Fig. 3 that $\bar{I}_{3}$ properly incorporates all two-particle thresholds at low energies and does not pick up any new low-energy endpoint singularities on the physical sheet. 4 It is also important to note that $\bar{I}_{3}$ receives no

3 For a review on Landau equations see Refs. [8-10] and references therein.

4 In particular, the pseudo-thresholds $P_{i}^{2}=(M-m)^{2}$ located at $( \pm 1,-m /(M-m))$ and indicated by $\circ$ in Fig. 3 are not promoted to thresholds on the physical sheet. 
additional contribution from the leading (i.e. pinch) singularity (dark shaded area in Fig. 3) other than that already present in $I_{3}$. This ensures that the analytic properties of $\bar{I}_{3}$ and $I_{3}$ coincide in the low-energy domain and implies that $R_{3}$ is analytic there.

By virtue of Eqs. (19) and (21) the regular part is

$$
R_{3}=\kappa_{3} \int_{1}^{\infty} \mathrm{d} z\left(\int_{-\infty}^{-1} \mathrm{~d} w+\int_{1}^{\infty} \mathrm{d} w\right) z[C(w, z)-\mathrm{i} \epsilon]^{d / 2-3} .
$$

The dependence on fractional powers of $M$ stems from the corner points located at $( \pm 1,1)$ (indicated by $\otimes$ in Fig. 3 ) and is entirely contained in $R_{3}$. It is instructive to verify this dependence on fractional powers of $M$ explicitly. The denominator in Eq. (23) is non-vanishing and of zeroth order in the low energy expansion in the whole integration domain and can, therefore, be expanded in $m^{2}$ and the small variables $s_{+}-s, P_{i}^{2}-M^{2}$ :

$$
C(w, z)=M^{2} z^{2} w^{2}+\mathcal{O}\left(s_{+}-s ; P_{i}^{2}-M^{2} ; m^{2}\right)
$$

The leading contribution in an expansion of $R_{3}$ is then

$$
\begin{aligned}
R_{3} & =2 \kappa_{3} M^{d-6} \frac{1}{d-4} \frac{1}{d-5}(1+\cdots) \\
& =-\frac{1}{16 \pi^{2}} \frac{1}{2 M^{2}}\left(\frac{2}{4-d}-2-\gamma-\log \frac{M^{2}}{4 \pi \mu^{2}}\right)(1+\cdots)
\end{aligned}
$$

where the omitted terms are analytic in the small quantities. The reason why $R_{3}$ has an ultraviolet divergence despite $I_{3}$ being ultraviolet finite is that in order to contain the fractional powers of $M$ while being regular in the lowenergy domain, it must include the appropriate logarithmic dependence on $\mu$, and this can only emerge if there is a $1 /(d-4)$ singularity.

The low-energy parts are shown below for $d \rightarrow 4$. The integral $\bar{I}_{3 A}$ is given by:

$$
\bar{I}_{3 \mathrm{~A}}=-\frac{1}{16 \pi \sqrt{s}} \int_{0}^{1} \mathrm{~d} z \frac{1}{\sqrt{C_{0}}}
$$

with

$C_{0}=z M^{2}+(1-z) m^{2}-\frac{z^{2} s}{4}-\frac{1}{2} z(1-z)\left(P_{1}^{2}+P_{2}^{2}\right)-\frac{(1-z)^{2}}{4 s}\left(P_{1}^{2}-P_{2}^{2}\right)^{2}-\mathrm{i} \epsilon$. 


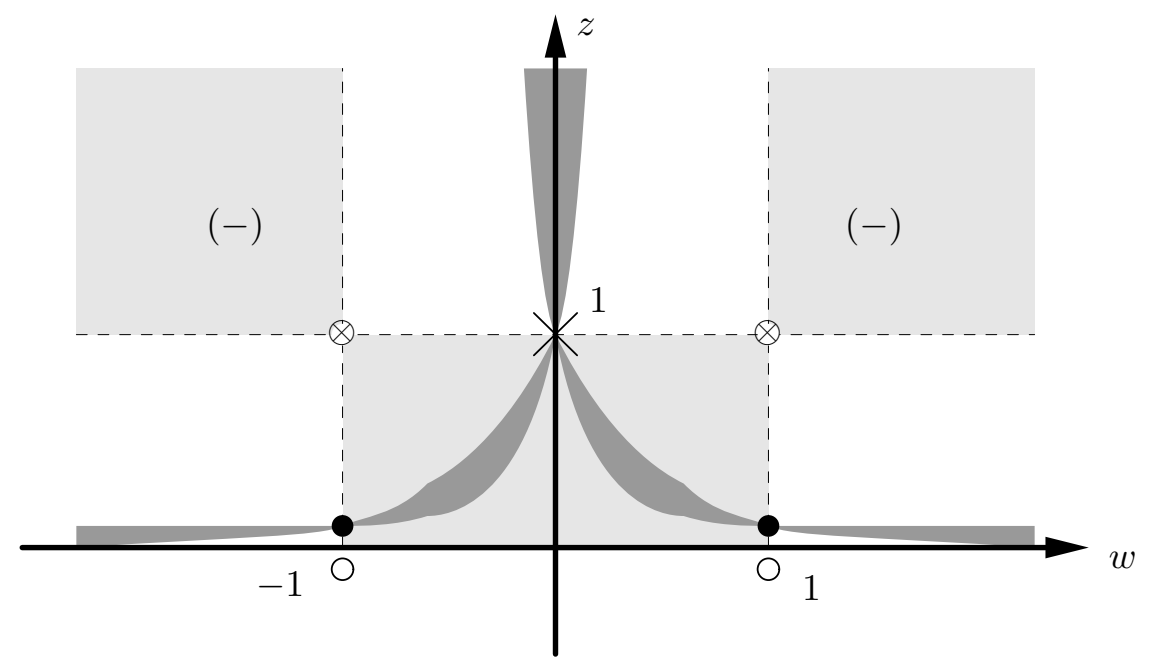

Fig. 3. Triangle diagram: Integration domain and location of singularities in the $(w, z)$-plane. The light shaded domains indicate the contour of integration of the regularized part $\bar{I}_{3}$, areas marked with $(-)$ enter with negative sign. The dark shaded area denotes critical points associated with the leading singularity of the triangle diagram. The two-heavy-particle $(\times)$ and the two heavy-light $(\bullet)$ thresholds lie on the boundary of the original integration domain $[-1,1] \times[0,1]$. Also indicated are the heavy-light pseudo-thresholds $(\circ)$. The corner singularities $(\otimes)$ give rise to fractional powers of $M$.

and the integral $\bar{I}_{3 B}$ is on the other hand given by:

$$
\begin{aligned}
\bar{I}_{3 \mathrm{~B}}= & \frac{1}{16 \pi^{2}}\left\{\left(\frac{2}{4-d}-\gamma-\log \frac{m^{2}}{4 \pi \mu^{2}}\right)\left(\frac{2}{s}+\cdots\right)\right. \\
+ & \left.\frac{1}{2}\left(\int_{-\infty}^{-1} \mathrm{~d} w+\int_{1}^{\infty} \mathrm{d} w\right) \frac{z_{0}}{\sqrt{D_{0} D_{1}}}\left(\frac{\pi}{2}+\arctan \left(\sqrt{\frac{D_{1}}{D_{0}}} z_{0}\right)\right)\right\},
\end{aligned}
$$

with

$$
\begin{aligned}
z_{0} & =\frac{1}{4 D_{1}}\left(2\left(m^{2}-M^{2}\right)+(1+w) P_{1}^{2}+(1-w) P_{2}^{2}\right) \\
D_{1} & =\frac{1}{2}\left((1+w) P_{1}^{2}+(1-w) P_{2}^{2}-\left(1-w^{2}\right) \frac{s}{2}\right), \\
D_{0} & =m^{2}-\frac{1}{4 D_{1}}\left(M^{2}-m^{2}-\frac{1}{2}(1+w) P_{1}^{2}-\frac{1}{2}(1-w) P_{2}^{2}\right)^{2}-\mathrm{i} \epsilon .
\end{aligned}
$$

It is to be noted that the integral $\bar{I}_{3 A}$ has no ultraviolet divergences. Such divergences can only come from the boundary contributions in the integration by parts over $w$, which in this case have to vanish because the domain of integration extends to the entire real axis. The low energy parts do not contain any fractional powers of $M$, as their explicit evaluation reveals the absence of $\log M^{2}$ terms. Thus, as in the case of the bubble diagram, the separation into low-energy and regular parts is also possible in the triangle diagram. The 
general procedure is now clear and can be easily carried over to other diagrams. As an illustration, we show this for the box diagram.

\subsection{The box diagram}

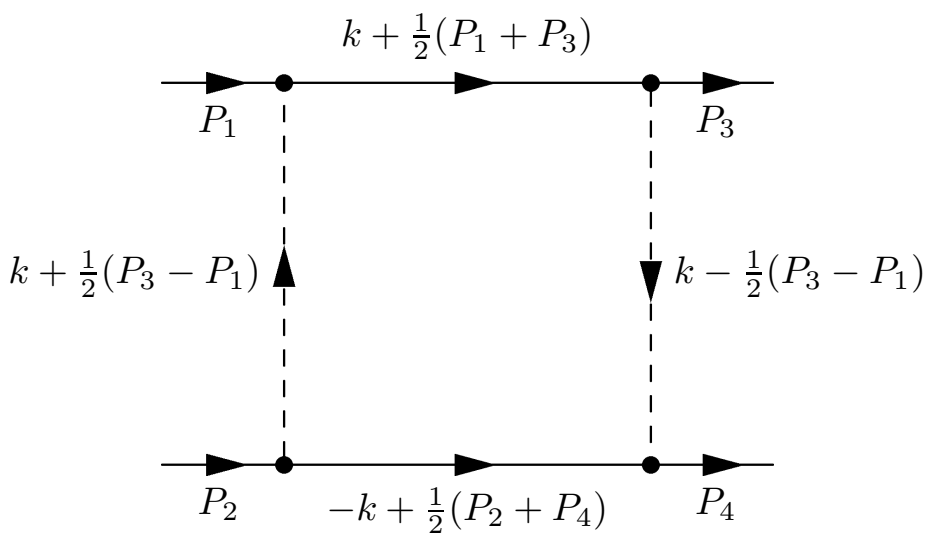

Fig. 4.

The scalar integral for the box diagram in Fig. 4 is

$$
\begin{aligned}
I_{4}=\mu^{4-d} & \int \frac{\mathrm{d}^{d} k}{(2 \pi)^{d}} \frac{\mathrm{i}}{\left(\frac{P_{1}+P_{3}}{2}+k\right)^{2}-M^{2}+\mathrm{i} \epsilon} \frac{\mathrm{i}}{\left(\frac{P_{2}+P_{4}}{2}-k\right)^{2}-M^{2}+\mathrm{i} \epsilon} \\
& \times \frac{\mathrm{i}}{\left(\frac{P_{3}-P_{1}}{2}-k\right)^{2}-m^{2}+\mathrm{i} \epsilon} \frac{\mathrm{i}}{\left(\frac{P_{3}-P_{1}}{2}+k\right)^{2}-m^{2}+\mathrm{i} \epsilon} .
\end{aligned}
$$

The light particle propagators can be combined to yield:

$$
\begin{aligned}
I_{4}=\mu^{4-d} \frac{\partial}{\partial m^{2}} & \int_{0}^{1} \mathrm{~d} x \int \frac{\mathrm{d}^{d} k}{(2 \pi)^{d}} \frac{1}{[k-\bar{q}(x)]^{2}-\bar{m}^{2}(x)+\mathrm{i} \epsilon} \\
& \times \frac{1}{\left(\frac{P_{1}+P_{3}}{2}+k\right)^{2}-M^{2}+\mathrm{i} \epsilon} \frac{1}{\left(\frac{P_{2}+P_{4}}{2}-k\right)^{2}-M^{2}+\mathrm{i} \epsilon}
\end{aligned}
$$

with

$$
\bar{q}(x) \equiv \frac{1}{2}\left(P_{3}-P_{1}\right)(1-2 x) \quad \text { and } \quad \bar{m}(x) \equiv m^{2}-\left(P_{3}-P_{1}\right)^{2} x(1-x) .
$$

Both $\bar{q}(x)$ and $\bar{m}(x)$ are small throughout the range of integration of $x$. Thus, the momentum integral is seen to be analogous to the triangle diagram. The procedure for separating $I_{4}$ into its regular and low-energy parts, $I_{4}=\bar{I}_{4}+R_{4}$, is therefore identical to the prescription given for the triangle diagram in the previous sub-section. 


\section{Discussion}

It has been shown that each basic one-loop integral $I$ in the two-heavy-particle sector can be decomposed into a low-energy part $\bar{I}$ whose low-energy analytic structure coincides with that of $I$, and a regular part which consists of the terms in $I$ that have factors of fractional powers of the large mass $M$. The EFTDR is now implemented by discarding $R$, which amounts to removing specific local terms in the low-energy domain. The EFTDR so defined is a natural regularization.

The local terms in $\bar{I}$ can be ordered according to a power counting scheme in the low-energy scales generically denoted by $p$. For a given one-loop diagram the leading order term is of order $p^{\nu}$ with $\nu=d-2 n_{\ell}-n_{\mathrm{h}}+n_{\mathrm{n}}$, where $n_{\ell}$ and $n_{\mathrm{h}}$ are respectively the numbers of light and heavy propagators, and $n_{\mathrm{n}}$ is the number of factors of small quantities in the numerator. This power counting is the standard one obtained in the $1 / M$ expansion [11].

The different non-analytic terms can be classified according to their infrared behavior. In particular, some of them are more singular than the local terms. For example, the low-energy power counting for the bubble diagram would be $\nu=2$, but Eq. (10) shows that it is more singular than this. If one takes $s-4 M^{2}$ to be of order $p^{2}$, the bubble diagram is of order $p$. In the case of the triangle, $\nu=0$, while $\bar{I}_{3 \mathrm{~A}}$ is more singular by one unit. As it is well known, these more singular behaviors are associated with the existence of the two-heavy-particle threshold.

As first noticed in reference [7], the fact that the low-energy and regular parts have different behaviors in $M$ (the regular part being entirely of fractional power of $\mathrm{M}$ and the low-energy part having no such terms), implies that the symmetries respected by dimensional regularization are also respected by each part separately and, therefore, by EFTDR.

Finally, the generalization to diagrams that involve insertions of small external momenta can be carried out quite easily. First, the propagators along each heavy particle line are separately combined using two sets of Feynman parameters, and a parameter $w$ is then used to combine the two results. Next, and after combining the light particle propagators, a second parameter $z$ is used to combine this result with that of the heavy propagators. The integration over the loop momentum is then performed, and subsequently the integration domain in $(w, z)$ is extended as in the case of the triangle diagram, leaving the integration domains of the remaining Feynman parameters unchanged. A more extensive presentation of this and other points in this letter will be given elsewhere [12]. 


\section{Acknowledgements}

This work was supported by the Department of Energy through contract DEAC05-84ER40150 (JLG), by the National Science Foundation through grants

\# PHY-9733343 (JLG and JS) and \# HRD-9633750 (DL and GP), by the Deutsche Forschungemeinschaft through project Le 1189/1-1 (DL), and by sabbatical leave support from the Southeastern Universities Research Association (JLG).

\section{References}

[1] S. Weinberg, Physica A96 (1979) 327.

[2] J. Gasser and H. Leutwyler, Ann. Phys. 158 (1984) and Nucl. Phys. B250 (1985) 465.

[3] J. Gasser, M. Sainio, and A. Švarc, Nucl.Phys. B307 (1988) 779.

[4] E. Eichten and B. Hill, Phys. Lett. B234 (1990) 511.

H. Georgi, Phys. Lett. B240 (1990) 447.

B. Grinstein, Nucl. Phys. B339 (1990) 253.

E. Jenkins and A. M. Manohar, Phys. Lett. B255 (1991) 558.

[5] V. Bernard, N. Kaiser, and U.-G. Meißner, Int. J. Mod. Phys. E4 (1995) 193, hep-ph/9501384.

[6] P. J. Ellis and H.-B. Tang, Phys. Rev. C57 (1998) 3356, hep-ph/9709354.

[7] T. Becher and H. Leutwyler, Eur. Phys. J. C9 (1999) 643, hep-ph/9901384.

[8] R. J. Eden, P. V. Landshoff, D. I. Olive, and J. C. Polkinghorne, The Analytic $S$-Matrix (Cambridge University Press, Cambridge, 1966).

[9] C. Itzykson and J.-B. Zuber, Quantum Field Theory (McGraw-Hill, New York, 1980).

[10] J. D. Bjorken and S. D. Drell, Relativistic Quantum Fields (McGraw-Hill, New York, 1965).

[11] S. Weinberg, Nucl. Phys. B363 (1991) 3.

[12] J. L. Goity, D. Lehmann, G. Prézeau, and J. Saez, in preparation. 